\title{
La finitude radicale de la vie
}

Réflexions à partir de la phénoménologie de Renaud Barbaras

\section{Raphaël Gély}

\section{(2) OpenEdition}

\section{Journals}

Édition électronique

URL : http://journals.openedition.org/alter/865

DOI : $10.4000 /$ alter.865

ISSN : 2558-7927

Éditeur :

Association ALTER, Archives Husserl (CNRS-UMR 8547)

\section{Édition imprimée}

Date de publication : 1 novembre 2013

Pagination : 239-253

ISBN : 978-2-95-223749-9

ISSN : 1249-8947

Référence électronique

Raphaël Gély, "La finitude radicale de la vie », Alter [En ligne], 21 | 2013, mis en ligne le 01 juin 2019, consulté le 10 octobre 2019. URL : http://journals.openedition.org/alter/865 ; DOI : 10.4000/alter.865 


\section{LA FINITUDE RADICALE DE LA VIE. RÉFLEXIONS À PARTIR DE LA PHÉNOMÉNOLOGIE DE RENAUD BARBARAS}

Raphaël Gély

L'objectif de cette contribution est de montrer de quelle façon la phénoménologie de Renaud Barbaras permet de faire droit à ce que l'on peut définir comme la finitude radicale de la manifestation. Si, chez Barbaras, la finitude constitutive de toute manifestation est radicale, c'est dans la mesure où elle est elle-même finie, c'est-à-dire habitée par une indétermination originaire. L'inachèvement constitutif de toute manifestation est lui-même inachevé. Il y a un polymorphisme de la finitude même de la manifestation. On trouve en ce sens chez Barbaras un dépassement de toute forme d'opposition abstraite entre présence à soi et absence à soi ou encore entre coïncidence avec soi et non-coïncidence avec soi. S'il y a, chez Barbaras, une non-coïncidence avec soi qui est constitutive de la moindre manifestation, cette noncoïncidence est elle-même en impossible coïncidence avec soi. C'est d'une non-coïncidence de la non-coïncidence qu'il est ici question. On ne peut correctement saisir les recherches que Barbaras réalise sur la vie du monde, la vie des vivants et la vie des humains si l'on ne fait pas droit à cette thèse centrale qu'il y a une finitude de la finitude, un polymorphisme de la finitude ${ }^{1}$. Il faut à cet égard noter que la façon dont Barbaras lit les auteurs qu'il mobilise dans la construction de sa

\footnotetext{
${ }^{1}$ Sans l'engager d'aucune manière dans ce qui va être ici exposé, c'est à Augustin Dumont que je dois de parler d'une finitude de la finitude, d'une finitude interne à l'épreuve que le fini fait de ce dont il est le fini. Cf. A. Dumont, L'opacité du sensible chez Fichte et Novalis. Recherches sur les théories et pratiques de l'imagination transcendantale dans leur mise à l'épreuve du langage, Grenoble, Millon, 2012, les chapitres II, III, IV.
} 
problématique consiste à faire de leur conflit le révélateur d'un débat interne à l'expérience elle-même. Il n'est donc plus question ici de faire de la non-coïncidence avec soi de la manifestation quelque chose de positif, qui serait comme tel sans conflit constitutif. Nous verrons comment cette attention du phénoménologue à l'indétermination profonde de la finitude même de la manifestation lui permet de développer une phénoménologie de la vie tout à fait originale, qui fait droit à la finitude radicale de celle-ci, à son impossible réduction à telle ou telle de ses figures. Au lieu de dire que l'expérience est nécessairement ouverte, en incessant devenir, parce qu'elle est finie - la question étant alors de déterminer de façon positive en quoi consiste sa non-coïncidence avec elle-même -, la radicalisation de l'interrogation phénoménologique réalisée par Barbaras consiste au contraire à faire droit au caractère lui-même polymorphique de cette finitude de l'expérience. La question est bien entendu alors de savoir comment faire droit à une telle finitude de la finitude même de la manifestation, comment réaliser des descriptions phénoménologiques ajustées à ce qui de la finitude de la manifestation est radicalement fini. La deuxième hypothèse de cet article est que les différents moments de la pensée de Barbaras, dont les moments dits cosmologique et métaphysique, peuvent être compris comme autant d'étapes dans l'approfondissement de cette interrogation sur la finitude radicale de la manifestation. C'est ainsi que les différents sens que la vie revêt dans la phénoménologie de Barbaras - la vie ontogénétique du monde lui-même, la vie des vivants et la vie des humains - sont autant de dimensions à la fois mêmes et autres d'une même vie mais dont la finitude est elle-même abyssale. Loin en ce sens que Barbaras cherche, dans les moments cosmologique et métaphysique de ses derniers travaux, à donner enfin à la finitude constitutive de la vie sa détermination purement positive, son plein sens, il cherche tout au contraire à faire pleinement droit à l'abyssalité même de la vie, à sa finitude radicale.

La vie, chez Barbaras, est tout à la fois elle-même et en incessant écart avec elle-même. Elle se déploie dans différentes figures qui sont à la fois autres et non-autres. Si les hypothèses qui sont au départ de cette recherche sont justes, on doit pouvoir montrer de quelle façon les descriptions phénoménologiques que Barbaras réalise font droit à ce polymorphisme radical de la vie, à l'identité et à la différence de ses figures. Je propose dans un premier temps de reprendre certaines des thèses de Barbaras sur le désir constitutif de la vie des vivants, ces thèses étant tout à fait caractéristiques de ce que je cherche à dire en parlant d'une finitude de la finitude même de la vie. Nous verrons en effet qu'il n'est aucunement question pour Barbaras de réduire ce désir 
à l'une ou l'autre de ses figures ${ }^{2}$, mais au contraire $d^{\prime} y$ inscrire un débat indépassable. Dans un deuxième temps, je montrerai comment cette question se déploie au niveau de la vie perceptive, pour montrer ensuite en un troisième temps et en un quatrième temps comment la prise en compte de la finitude radicale de la manifestation exige le passage par ce que Barbaras caractérise avec Patočka et au-delà de ce dernier comme les moments cosmologique et métaphysique de sa phénoménologie.

\section{La finitude du manque}

Avant d'entrer dans des considérations plus spécifiquement liées à la question de la perception, je propose donc de reprendre certaines thèses de Barbaras sur le manque constitutif du désir, lequel est identifié à l'affectivité même de la vie des vivants. Selon l'hypothèse directrice de cette recherche, il y a nécessairement chez Barbaras un manque au cœur même du manque, un manque du manque comme réalité positive, comme réalité assurée de son sens, de ce dont il est le manque ou de ce que c'est pour un manque que d'être un manque. Chez Barbaras, la moindre affection, pour être la manifestation d'une vie intrinsèquement inépuisable, ne peut manquer "d'être creusée d'une distance intérieure, marquée par l'impossibilité de s'étreindre elle-même $»^{3}$, c'est-à-dire de coïncider avec la puissance de vie qui l'habite. L'affectivité intrinsèque du vécu implique l'épreuve d'un manque au cœur même de ce vécu. Pour Barbaras, l'épreuve de ce manque n'est pas une épreuve parmi d'autres possibles, mais ce qui rend possible toute épreuve comme épreuve : «[...] c'est seulement parce qu'elle est vie du désir que la Vie est affectivité $»^{4}$. Mais il faut encore ajouter que cette épreuve est elle-même en manque de son propre sens, est en débat avec soi. C'est à ce titre, me semble-t-il, que le manque est, chez Barbaras, au fondement même de l'affectivité originaire de la vie. Il ne peut être question ici d'opposer à l'épreuve originaire d'une pure présence à soi l'épreuve originaire d'un manque de soi si l'on ne saisit pas que ce manque est lui-même en

\footnotetext{
2 Pour cette hypothèse d'une pluralité irréductible des figures de la finitude constitutive de la manifestation, je me permets de renvoyer également à $R$. Gély, «Du retard de la conscience à la facticité du monde. Réflexions à partir des recherches de Daniel Giovannangeli (Introduction) ", in D. Giovannangeli, Figures de la facticité. Réflexions phénoménologiques, Bruxelles, PIE Peter Lang, 2010, p. 11-50.

${ }^{3}$ R. Barbaras, Introduction à une phénoménologie de la vie, Paris, Vrin, 2008, p. 299.

${ }^{4}$ Ibid., p. 300.
} 
manque d'un sens achevé, est habité par des dimensions à la fois mêmes et autres. C'est ainsi encore que Barbaras parle d'une différence pure, d'une différence qui ne va pas jusqu'au bout d'elle-même, d'une différence dont le mouvement même de différer se retient en lui-même, ne s'accomplit qu'en ne s'accomplissant pas : «Il faut enfin souligner que cette privation n'est autre qu'une interruption ou un suspens et qu'elle n'a donc même pas, pour ainsi dire, la positivité d'un défaut. Ici, la privation ne correspond pas au surgissement d'une négativité et la différence humaine est en ce sens une différence pure $»^{5}$. Ainsi, jamais, le manque ne peut-il être déterminé de façon positive, ou encore coïncider avec lui-même. C'est pour cette raison que l'épreuve originaire du manque interne à la moindre affection est tout autant l'épreuve d'une plénitude, d'une présence excessive, d'une présence qui n'est la présence de rien de déterminé, que l'épreuve d'une absence excessive, c'est-à-dire d'une absence qui est absence de rien de déterminé.

Il est en effet caractéristique de la phénoménologie de Barbaras de qualifier le désir tout autant comme insatisfaction absolue que comme sérénité, désir d'accueillir tout ce qui peut se donner, « disponibilité » originaire. L'affectivité originaire de la vie est liée à l'excès de la puissance d'éprouver sur chaque affection, à l'épreuve que chaque affection fait d'elle-même comme désir qu'il y ait toujours et autrement de la vie. C'est pour cette raison que l'épreuve du manque est tout autant sérénité, désir d'accueillir tout ce qui peut se donner, « disponibilité » originaire ${ }^{6}$. On trouve ainsi chez Barbaras cette thèse très forte d'un désir qui, originairement incomblable, originairement désir de rien de déterminé, peut s'éprouver en même temps comme plénitude de chaque instant. Cette inépuisable activité du désir originaire de la vie, de son désir d'altérité, est identiquement son pouvoir de pâtir de tout ce qui advient, d'accueillir le donné pour luimême et pour tout ce qu'il ouvre comme possible. Le Désir ainsi compris est «la forme même de la réceptivité »7. On trouve dans cette puissance sans fin du désir la condition du pâtir, la possibilité pour le vivant d'être affecté en profondeur par ce qui lui advient. Chez Barbaras tout comme chez M. Henry mais selon une autre modalité, il n'y aucune indifférence possible du pouvoir d'éprouver par rapport à ce qui est à chaque fois éprouvé : «En tant qu'elle est Désir, la vie est

${ }^{5}$ Ibid., p. 242.

${ }^{6}$ Ibid., p. 300.

7 Ibid., p. 301. 
le lieu de tout sentir et de toute épreuve : elle est Affectivité $»^{8}$. Une activité originaire est au cœur même de l'ipséité de l'affection : «Bref, comme agir non qualifié, aspiration pure, le désir est identiquement pâtir ; son activité est pure passivité, ou plutôt, il est l'identité réalisée de l'activité et de la passivité ${ }^{9}$. Sans cesse, il s'agit chez Barbaras d'interroger le désir comme l'identité même de dimensions tout à la fois différentes et non différentes, de différences qui n'en sont pas, de différences des identiques. Ce que Barbaras cherche à interroger, c'est précisément l'impossibilité de subordonner une figure du désir à l'autre, ce qui suppose qu'on puisse les décrire comme étant tout à la fois autres et non-autres, comme étant autres en tant que non-autres. L'affectivité radicale de la vie comme désir renvoie à une finitude indépassable du sens même de la finitude, à une finitude qui exige pour être pensée en sa radicalité l'articulation de différentes épreuves, à la fois mêmes et autres, de la vie. La moindre affection, en sa texture affective originaire, se déploie donc selon des dimensions qui sont tout à la fois mêmes et autres. Pour approfondir cette question, je propose de reprendre certaines des thèses de Barbaras sur le caractère perspectiviste $\mathrm{du}$ perçu.

\section{Les lacunes du perçu}

Comme on le sait, dès ses recherches sur Merleau-Ponty, Barbaras prend au sérieux et radicalise cette thèse selon laquelle la chose perçue se donne en se retirant et se retire en se donnant: "L'invisibilité du sens ne signifie donc pas que le sensible, par lequel elle se réalise, soit d'un autre ordre que celui du sens, écrit Barbaras: le sensible le présente comme une "certaine absence" $\gg 10$, cette absence n'étant que l'envers de la possibilité que ce sens a de se donner ailleurs et autrement tout aussi pleinement qu'ici et maintenant. Ce qui ressort de l'examen phénoménologique le plus simple de l'épreuve perceptive du sens d'une réalité donnée, c'est que celle-ci, en sa phénoménalité même, est porteuse d'un sens que tout à la fois elle possède et partage, ce qui signifie que toute réalité particulière perçue est habitée par une possibilité d'apparaître qui outrepasse de façon nécessaire son mode actuel de donation. La question est alors de

\footnotetext{
${ }^{8}$ Idem.

${ }^{9}$ Ibid., p. 300.

${ }_{10}$ R. Barbaras, De l'être du phénomène. Sur l'ontologie de Merleau-Ponty, Grenoble, Millon, 1991, p. 191.
} 
saisir de quelle façon cette tension entre l'immanence du sens au perçu et la transcendance du sens au perçu, sa visibilité et son invisibilité, se déploie. Dans le cadre de l'interrogation développée ici, il faut montrer que l'épreuve de ce même profil est, comme telle, à la fois et dynamiquement, épreuve d'une présence et épreuve d'une absence. Nous verrons que chez Barbaras la présence dont il est ultimement fait l'épreuve en ce profil, ou à un autre niveau en cette chose ou encore en cette situation, est celle de la puissance ontogénétique du monde, de la même façon que l'absence dont le pâtir de ce profil, de cette chose, de cette situation, etc., est l'épreuve même, est une absence radicale, une absence qui n'est pas celle d'un objet déterminé, mais celle du monde lui-même.

Il revient à la phénoménologie de Barbaras de prendre au sérieux cette thèse selon laquelle la chose même se donne en chacun de ses aspects de la même façon que c'est le monde en sa puissance inépuisable d'être, de détermination, qui se donne en chaque chose. Nous avons toujours affaire chez Barbaras à des mouvements qui se déploient à l'intérieur de mouvements. Ainsi, ce rouge est manifestation de ce coquelicot et ne peut l'être qu'en donnant à éprouver une puissance d'être qui, en définitive, est celle du monde lui-même, du monde compris comme source, comme puissance d'être en incessante effectuation de soi. En ce sens, le rouge de ce coquelicot ne peut être la manifestation même de ce coquelicot que si ce coquelicot rouge est ce en quoi et comme quoi la puissance ontogénétique de l'univers se déploie, se détermine, se manifeste. Percevoir ce coquelicot, le percevoir comme tel en cet aspect-ci de lui-même, c'est nécessairement chez Barbaras, non pas le manifester, mais recueillir cette manifestation, cette détermination qu'il est en sa matérialité même : «La subjectivité est essentiellement mouvement car son acte de synthèse vient recueillir une synthèse déjà effectuée dans les choses et prolonge ainsi le mouvement ontologique dont cette synthèse est l'œuvre $»^{11}$. Percevoir ce coquelicot rouge, c'est pâtir de cette puissance de manifestation, de détermination de soi, que le monde est dynamiquement en lui et comme lui. Il ne peut en ce sens être question de se contenter d'affirmer que la synthèse perceptive renvoie à une synthèse déjà réalisée dans le perçu lui-même si l'on ne saisit pas que cette synthèse matérielle renvoie à une dynamique cosmologique, à une puissance

\footnotetext{
${ }^{11}$ R. Barbaras, Le mouvement de l'existence. Études sur la phénoménologie de Jan Patočka, Chatou, Les Editions de la Transparence, 2007, p. 77.
} 
inépuisable de détermination ${ }^{12}$. Ainsi, la chose ne détient cette puissance inépuisable d'être chacune de ses déterminations que de la puissance même de l'univers qui s'y détermine, s'y déploie.

La chose se déploie en ses qualités et ce déploiement est un point de passage du déploiement même du cosmos. Si la moindre chose renvoie, en étant perçue, à tant d'autres choses effectives ou possibles, c'est tout d'abord parce qu'elle est comme telle une manifestation, c'est-à-dire ce en quoi et comme quoi le fond de l'Etre, en sa puissance d'être un cosmos, se détermine, sort de son indifférenciation originaire ${ }^{13}$. Si la qualité de la chose est présence de la chose, $c^{\prime}$ est parce que le monde $s^{\prime} y$ produit, $s^{\prime} y$ détermine, sort de son indifférenciation originaire à la façon d'un rouge, à la façon d'un coquelicot rouge, à la façon de ce coquelicot qui est rouge. Il faut ainsi prendre toute la mesure de cette thèse selon laquelle, au cœur même de la perception de ce coquelicot, une puissance inépuisable d'être est éprouvée, est recueillie. Il y a au cœur de la perception de ce coquelicot l'épreuve comme telle non intuitive, l'épreuve affective, d'une présence excessive, d'une présence de rien de déterminé. Ce coquelicot est, en sa détermination même, manifestation d'un pouvoir inépuisable de se manifester. En sa limite, c'est un pouvoir sans limite de se limiter qui se déploie. La moindre limite est, de ce point de vue, non épreuve de ce qui n'est pas suffisamment là, mais épreuve de ce qui est excessivement là. Si la puissance d'être de l'univers se comprend d'un point de vue hénologique comme une puissance de détermination de soi, de différenciation et d'unification de soi, il nous faut comprendre toute limite, toute manifestation, comme passage d'un infini, comme ce en quoi et comme quoi une puissance inépuisable d'être se met en mouvement, se détermine. Nous dirons ainsi que ce rouge ne peut être la manifestation d'un coquelicot, être ce coquelicot, que parce qu'il n'y a de coquelicot que généré dans la dynamique inépuisable d'une ontogenèse matérielle. Si ce rouge est manifestation du coquelicot, c'est parce qu'il n'y a de coquelicot que comme point de passage d'une puissance inépuisable de détermination de soi. C'est de cette façon que je propose de comprendre cette thèse de Renaud Barbaras, d'une extrême profondeur, selon laquelle le «fini n'est pas négation de l'infini, car cette dimension d'excès n'a aucune positivité et n'a donc pas d'autre

\footnotetext{
${ }_{12}$ Cf. R. Barbaras, L'ouverture du monde. Lecture de Jan Patočka, Chatou, Les Editions de la Transparence, 2011, p. 223-253.

${ }_{13}$ Pour la question de la différence hénologique comme différence phénoménologique, cf. par ex. R. Barbaras, Le mouvement de l'existence, op. cit., p. 45-60.
} 
réalité que celle du fini qu'elle excède toujours $»^{14}$. Le fini est le fini d'un infini qui s'y accomplit lui-même, y prend possession de luimême. C'est dire que la moindre qualité d'une chose, la moindre chose, etc., est, aussitôt, sans médiation, épreuve affective d'une inépuisabilité.

Barbaras refuse d'accorder au monde une plénitude que l'on pourrait qualifier de substantielle. Il est impératif, pour faire droit à la donation même des choses en leurs qualités, d'accorder au monde lui-même une certaine faiblesse ou incomplétude ontologique. Plus profond que le monde des étants, il y a une "mondification », c'est-àdire, écrit Barbaras, " un devenir-monde du monde, une auto-constitution du monde sous la forme précisément de l'incessant avènement des étants en son sein ${ }^{15}$. Le mouvement du monde n'est pas un mouvement ontique. C'est un mouvement hors du fond, ce qui suppose l'incessant surgissement de ce mouvement : «Certes pas de fond sans mondification, écrit encore Barbaras, mais incessante survenue de ce mouvement ${ }^{16}$. C'est précisément parce que le mouvement de mondification du monde est toujours encore pour une part en retrait par rapport à lui-même, ne cesse de surgir, que chaque étant peut, en sa limite même, être éprouvé comme un point de passage du monde, comme le passage même du monde, comme une mise en mouvement du monde en l'inépuisabilité même de son pouvoir de se mouvoir.

On trouve chez Barbaras cette thèse très forte, notamment développée dans sa lecture patočkienne d'Aristote, qu'il y a une nécessaire limitation interne du processus même de limitation. Pour s'accomplir, le processus de limitation doit être pour une part retenu. Il y a une finitude de la finitude à tout niveau, et cela se traduit sur le plan ontogénétique par ce que l'on peut caractériser comme une limitation de la limitation, comme une retenue intérieure au mouvement de limitation. Le mouvement naturel du monde ne se donne absolument en chaque étant qu'en s'y limitant. Le rouge de ce coquelicot n'est absolument ce coquelicot que dans la mesure où il ne l'est pas absolument, que dans la mesure où il est ouvert de l'intérieur de lui-même à d'autres manifestations tout aussi absolues de ce même coquelicot. C'est à tout niveau de la vie de la manifestation que ce mouvement de limitation et de limitation de la limitation se produit. Chaque qualité n'est une qualité absolue parmi d'autres qualités absolues qu'en étant qualité de choses qui sont elles-mêmes autant de

\footnotetext{
${ }^{14}$ R. Barbaras, Introduction à une phénoménologie de la vie, op. cit., p. 280.

${ }^{15}$ R. Barbaras, La vie lacunaire, Paris, Vrin, 2011, p. 103.

16 Idem.
} 
manifestations absolues d'une même puissance inépuisable d'être. Il est de la plus grande importance de bien saisir que c'est précisément parce que le mouvement ontogénétique du cosmos s'initie, ne cesse de s'initier, dans la détermination de cette chose-ci tout autant que dans la détermination de cette chose-là, que la moindre épreuve perceptive est chez Barbaras le recueil du mouvement même du monde, du monde dans l'inépuisabilité de son incessante mise en mouvement de soi.

Mais il faut alors aussitôt ajouter, c'est le second temps de cette analyse, que ce coquelicot rouge éprouvé comme le devenir-rouge $\mathrm{d}$ 'une puissance inépuisable $\mathrm{d}^{\prime}$ être est tout aussi radicalement éprouvé comme la présence même d'une absence radicale, d'une absence de rien de déterminé. Il y a au cœur même de la perception de la chose, indissociable de cette perception, l'épreuve d'une absence radicale. C'est, me semble-t-il, une originalité profonde de la phénoménologie de Barbaras que de faire de l'éprouver même de la chose perçue l'éprouver d'une absence radicale. La chose perçue est, en et comme sa donation même, donation d'une absence radicale. La chose se donne à la fois comme le recueil d'une puissance inépuisable d'être et comme le point de départ d'une quête sans fin. Il faut tenir ensemble ces deux dimensions de l'expérience perceptive, refuser de subordonner l'une à l'autre. Ce qui fait la dynamique de la vie perceptive, c'est tout autant l'épreuve en chaque perception d'un excès radical de présence que l'épreuve d'un excès radical d'absence. À chaque fois que l'on veut subordonner une dimension à l'autre, c'est le cour vivant de la vie de la manifestation, sa finitude radicale, qui est mis à mal. La moindre chose est ce dans la perception de quoi la puissance illimitable de l'Archi-Vie du monde se donne comme telle à éprouver. Mais il faut aussitôt ajouter que la moindre chose est ce dans la perception de quoi un manque radical, un désir incomblable, de rien, surgit. Nous dirons ainsi qu'en la perception même de ce coquelicot, une plénitude tout à la fois se donne et se retire, les deux à la fois, absolument, se donne absolument et se retire absolument. La perception de ce coquelicot rouge est identiquement épreuve d'une présence inépuisable, $d$ 'une présence de rien de déterminé, et épreuve d'une absence indépassable, d'une absence de rien de déterminé. 


\section{Les mouvements de la vie}

Les mouvements de la vie tels que Barbaras les décrit doivent être compris à partir de cette exigence première d'une attention à la finitude même de toute manifestation. Repartons du rouge de ce coquelicot. En ce rouge apparaissant, l'archi-mouvement du monde se donne comme tel à éprouver. Ce rouge, qui est comme tel une manifestation du monde, est recueilli, devient un rouge apparaissant à un vivant. En même temps, avons-nous vu, ce rouge est, comme tel, la donation d'une absence indépassable. En et comme sa limite même, dans le recueil de sa synthèse matérielle, ce coquelicot rouge est manifestation de ce qui est excessivement présent et manifestation de ce qui est excessivement absent. Pour rendre compte de cette indépassable tension intérieure au pâtir même de ce coquelicot rouge, il faut, dans la perspective de Barbaras, interroger le vivant, et tout d'abord tout vivant, dans l'épreuve qu'il fait à la fois de son pouvoir d'être affecté par la vie même du monde, de recueillir cette vie, et d'en être expulsé. Le vivant est, chez Barbaras, cet étant qui, produit par le mouvement du monde, ne peut manquer en même temps de s'éprouver expulsé hors de la vie même du monde. Il faut insister sur l'idée que le vivant est expulsé, non de ce qui est produit par le monde, non du plan des étants, mais du cœur vivant du monde. Le vivant ne peut être le vivant qu'il est, être en vie et éprouver quoi que ce soit, que parce qu'il survient, au sein même du monde, comme une exposition au mouve-ment même du monde. En la réception même du moindre étant, c'est la puissance même du monde qui est recueillie, dont il y a pâtir. Le vivant est cet étant inachevé mis en demeure, en acceptant d'une façon passive cet inachèvement, de faire être cet inachèvement, de l'empêcher de s'évaporer, de se dissiper, de le faire être en devenant lui-même, à l'image du monde comme tel, une puissance de mise en mouvement de soi, une puissance de sortie hors de son propre fond abyssal. Le vivant ne peut être affecté par quoi que ce soit qu'en y éprouvant la puissance d'être du monde. Il ne trouve l'énergie nécessaire pour endurer son inachèvement que parce qu'en celui-ci la vie du monde se donne à éprouver, se communique. Mais ce pouvoir de se laisser affecter en toute chose par la vie du monde, le vivant ne l'est qu'à s'éprouver en même temps expulsé de celle-ci, expulsé de cela même qui en même temps ne cesse de se communiquer à lui, de l'affecter $^{17}$.

\footnotetext{
17 Pour les remarquables descriptions de cette expulsion originaire, cf. R. Barbaras, L'ouverture du monde, op. cit., p. 282-294.
} 
Le pouvoir d'éprouver quoi que ce soit renvoie chez Barbaras à l'épreuve affective originaire du mouvement même du monde et de son expulsion hors de celui-ci. Le pouvoir de recueillir cette puissance inouie du monde en chaque chose est ce qui définit le pouvoir d'éprouver. Et ce pouvoir d'éprouver ne peut être ce pouvoir d'accueil en chaque chose du monde lui-même, de sa puissance, qu'en éprouvant son impuissance à être le monde, qu'en éprouvant son impuissance à rejoindre la puissance originaire du monde. Il ne s'agit donc en aucune manière chez Barbaras de dire que le vivant est expulsé de l'ordre des étants, du monde dans sa factualité. Ce dont le vivant est expulsé, ce n'est pas de ce que le monde produit. Le vivant est un étant parmi d'autres étants. Ce qui fait la caractéristique de cet étant est précisément qu'il s'éprouve expulsé du mouvement même $\mathrm{du}$ monde, de l'Archi-mouvement du monde, non pas, répétons-le, expulsé de ce qui est, mais, au contraire, expulsé du mouvement même de production de ce qui est, de la vie même du monde. Tout se passe, avec le vivant, comme si le mouvement même du monde sortait de soi, se trouvait expulsé, en un point, hors de lui-même ${ }^{18}$. Il $\mathrm{n}^{\prime} \mathrm{y}$ a pas, chez Barbaras, de sujet séparé, mais l'archi-événement d'une séparation, d'une séparation non hors de l'étant, mais hors du mouvement même du monde, hors de l'incessant mouvement de mondification du monde. Dans l'Archi-événement de la séparation du mouvement du monde d'avec lui-même, tout se passe comme si le mouvement même du monde échouait à produire des étants demeurant dans les limites qui leur sont imparties, comme si le vivant était cet étant à la fois trop et trop peu individué. Cet inachèvement permanent, le vivant qui y advient ne peut l'éprouver que comme une incessante expulsion hors du mouvement même du monde. Autrement dit, cet inachèvement ne devient celui d'un vivant possible que parce qu'il est éprouvé par celui-ci comme une expulsion hors de la vie du monde.

Il est de la plus grande importance de bien saisir que le vivant ne peut s'éprouver expulsé hors du mouvement de la manifestation du monde que parce qu'il en est l'épreuve comme telle, que parce qu'il pâtit en tout ce qui lui arrive de la vie même du monde, qu'il est affecté par elle. Le vivant, inachevé, ne peut être le vivant qu'il est, consentir créativement à sa vulnérabilité, qu'en étant d'emblée affecté en tout ce qui lui arrive par la puissance vertigineuse de l'automondification du monde. C'est à ces considérations profondément originales que Barbaras nous conduit. Le vivant, cet être en vie

${ }_{18}$ Cf. R. Barbaras, La vie lacunaire, op. cit., p. 156. 
s'éprouvant vivre, ne peut surgir dans le monde que comme ce pouvoir de pâtir, en toute chose, du mouvement même du monde. Ce qui est fondamentalement éprouvé en toute affection, c'est le mouvement même du monde, sa puissance inépuisable de mise en mouvement. Le vivant est cet étant qui pâtit en tout étant du monde lui-même. Il faut plus encore dire qu'il n'y a de pâtir possible, au sens $\mathrm{du}$ recueil, subjectif, de ce qui se manifeste, que pour un vivant s'éprouvant expulsé hors de la vie même du monde, et ne pouvant l'être qu'en pâtissant de cette vie du monde. Ce que le vivant perd en devenant le vivant qu'il est, c'est ce qu'il ne pourra jamais posséder, c'est ce qu'il désire à jamais, et cela à la mesure de son pouvoir de $\mathrm{s}^{\prime}$ en laisser affecter. Il est la perte de ce qu'il ne cesse, en toute chose, de recueillir, à savoir la vie même du monde, la puissance abyssale de son incessante mise en mouvement. Ce qui fait que cet étant parmi d'autres qu'est le vivant est en même temps fondamentalement autre que tout étant matériel, c'est qu'il s'éprouve, parce qu'il est expulsé hors de la vie du monde, apparenté au mouvement même du monde, à la vie même du monde.

$\mathrm{Au}$ sens fort, le vivant se meut. Il n'est pas seulement mû à la façon des étants non-vivants. Il ne s'éprouve pouvoir se mouvoir qu'à la mesure de l'épreuve qu'il fait, dans le pâtir même de l'être-mu des étants, du se-mouvoir même du monde. C'est dans un même geste donc que Barbaras refuse d'assimiler, en dernière instance, le vivant aux étants naturels du monde, et refuse de l'en dissocier. Ils sont à la fois autres et non-autres. Toute réduction de l'archi-mouvement du monde à un mouvement ontique ne peut en ce sens qu'occulter en profondeur le rapport de la vie des vivants à la vie même du monde, l'apparentement du pouvoir de se-mouvoir des vivants à la vie même du monde. La subjectivité de la vie est indissociable de son pouvoir d'éprouver, en toute chose, le mouvement même du monde et de s'en éprouver, pour cette raison même, expulsée. Le mouvement même du vivant est recueil et participation à la vie même du monde, à la vie de la manifestation. Mais il est tout autant épreuve d'une absence radicale, perte même de ce qui, sans cesse recueilli, ne peut être possédé.

\section{La privation humaine comme privation de la privation}

La question qu'il faut dès lors se poser est celle du rapport de la vie humaine à cette expulsion originaire hors de cette vie même du monde qui est constitutive de tout vivant. Dans la perspective de 
Barbaras, la vie de l'humain peut être comprise comme une privation redoublée, comme une privation au cœur même de cette privation de la vie du monde qu'est le vivant. L'objectif de ce dernier point est de montrer que le vivant humain peut être compris comme ce vivant qui fait l'épreuve elle-même radicalisée de la finitude radicale de la vie. Comme le montre remarquablement Barbaras, le propre du vivant animal est de convertir aussitôt l'épreuve de la vie de ce monde ainsi simultanément reçu et perdu en l'effectivité de mouvements désirants $^{19}$. L'archi-événement de l'expulsion du vivant hors de la vie du monde consent à soi, devient la vie d'un vivant aussitôt emporté dans l'inépuisabilité de sa propre puissance de désirer, c'est-à-dire de se mouvoir. L'animal, aussitôt, dans l'effectivité de son mouvement désirant, adhère à cette privation qu'il est, il y adhère selon la modalité précisément d'un mouvement sans fin possible. C'est ce qui fait dire à Barbaras que l'épreuve de la privation se réalise chez l'animal plutôt comme mouvement que comme épreuve ${ }^{20}$. La privation anthropologique peut en ce sens être comprise comme une privation de ce que la privation, en consentant à soi, rend immédiatement possible, à savoir l'effectivité de mouvements désirants. Dans la privation anthropologique, le suspens hors de l'Archi-vie du monde se retient pour une part en lui-même, ne se transforme pas aussitôt en l'effectivité de mouvements désirants, de sorte qu'avec l'humain l'archi-événement en lequel le vivant advient à lui-même pâtit comme tel de soi, se retient, s'intériorise.

$C^{\prime}$ est en ce sens que l'on peut reprendre les considérations fondamentales de Barbaras sur cette impuissance radicale qui est au cœur même du pouvoir de se mouvoir des humains ${ }^{21}$. Cette impuissance renvoie bien entendu à la prématuration profonde du corps humain à la naissance, mais elle est tout autant présente dans la façon même dont tout mouvement humain se retient pour une part, est habité par une retenue fondamentale, par une retenue constitutive de son mode propre d'ouverture au monde en tant que tel: "Ressaisie du point de vue du vivre, la conscience ne désigne rien d'autre que cette retenue, cette interruption ou ce suspens au sein du vivre, inhérente à son rapport toujours déçu, à l'Ouvert. La conscience n'est autre que ce rassemblement sur soi, cette réserve ou ce pli induits par le retard ou le retrait sur soi du vivre comme vivre dans l'Ouvert $»^{22}$.

\footnotetext{
${ }^{19}$ Cf., par ex. R. Barbaras, La vie lacunaire, op. cit., p. 158.

${ }^{20} \mathrm{Cf}$. Idem.

${ }^{21}$ Cf. R. Barbaras, Introduction à une phénoménologie de la vie, op. cit., p. 116.

22 Ibid., p. 261.
} 
Le vivant humain, en la retenue intérieure de son pouvoir de se mouvoir, peut se laisser affecter pleinement par ce qui se manifeste, se laisser affecter par l'étant dans la pleine densité de sa présence. Le vivant humain, en cette retenue intérieure, est consentement à pâtir absolument de l'indépassabilité même de ce qui ici et maintenant l'affecte. Il est consentement à pâtir, en tout ce qui se donne, de la puissance même du monde. Mais il faut aussitôt ajouter que ce vivant, ainsi immobilisé-s'immobilisant, exposé radicalement en toute chose à la puissance ontogénétique du monde, est tout autant, pour cette raison même, épreuve d'une insatisfaction radicale, épreuve d'une absence indépassable, désir de mouvement. C'est parce que le vivant humain advient tout à la fois comme celui qui pâtit en chaque chose d'une indépassable présence et d'une tout aussi indépassable absence qu'il lui est donné de pouvoir pâtir du sens même de ce qui est en train de l'affecter, des possibilités mêmes de ce qui l'affecte ${ }^{23}$. Chez l'animal, la chose perçue engage aussitôt un mouvement effectif au-delà d'elle-même, n'arrête pas absolument le regard. Chez le vivant humain, la chose perçue, tout à la fois, arrête absolument le regard et ouvre à un mouvement possible au-delà d'elle-même ou encore à une diversité de rapports possibles à elle-même. C'est du possible qu'il est donc ici question. C'est précisément parce que le vivant humain pâtit de sa propre expulsion hors de la vie animale et ce faisant pâtit absolument de son expulsion hors de la vie du monde qu'il peut tout à la fois pâtir de chaque chose en sa densité matérielle, en l'obstination de sa présence, et pâtir de ce qu'elle peut devenir. Entre l'épreuve de l'extrême densité de ce qui se manifeste et l'incessant mouvement effectif de son dépassement, il y a l'épreuve $\mathrm{du}$ possible en tant que possible. L'incessante retenue intérieure du vivant humain est en ce sens indissociable de son pouvoir, non seulement de se mouvoir effectivement, mais de faire l'épreuve de mouvements possibles, de potentialiser des mouvements. Un des apports, considérable, de la phénoménologie de Barbaras est de montrer que la question du possible renvoie nécessairement à la finitude radicale de la manifestation. C'est parce que la chose, en sa limite même, donne à la fois à éprouver la présence indépassable du monde et son absence tout aussi indépassable qu'elle se donne tout autant à éprouver en ses possibilités.

L'archi-événement de l'advenue du vivant hors de la vie même du monde ne cesse d'avoir lieu, ne cesse de se répéter au cœur même de la moindre affection. Ainsi, la perception de ce coquelicot est-elle tout

${ }^{23}$ Cf. R. Barbaras, L'ouverture du monde, op. cit., p. 267-282. 
autant, en sa finitude même, le recueil, dense absolument, de la vie du monde et l'épreuve, dense absolument, de sa perte irrémédiable. C'est bien au cœur de cette extraordinaire tension, qui fait de la finitude de l'apparaître une finitude radicale, que la question du possible et de son rapport à la liberté doit, chez Barbaras, être posée. La moindre expérience perceptive est, en sa texture affective radicale, le recueil possibilisant d'une puissance inépuisable d'être et le désir possibilisant de ce qui à jamais ne peut être possédé. La liberté se comprend désormais ici tout à la fois comme pouvoir d'arrachement au donné et comme pouvoir d'exposition au donné, comme exposition, en chaque chose, à la puissance même du monde et comme quête, à partir de chaque chose, de ce qui ne peut comme tel être possédé. Un des enjeux les plus fondamentaux de la phénoménologie de Barbaras est en ce sens de montrer de quelle façon la liberté, en son sens le plus profond, est indissociable de ce que l'on peut appeler la finitude radicale de la vie, la finitude même de la finitude. 